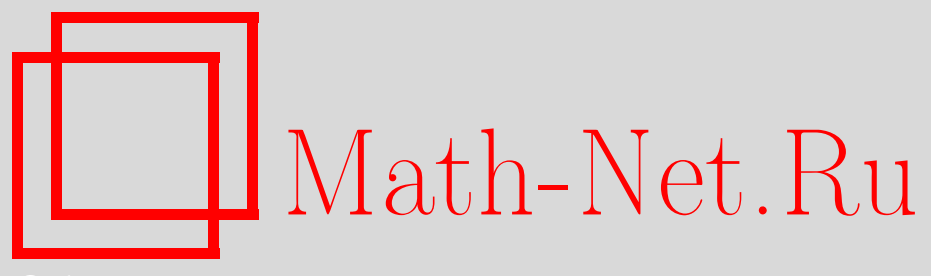

С. М. Ермакова, Векторные расслоения конечного ранга на полных пересечениях конечной коразмерности в линейном инд-грассманиане, Матем. заметки, 2015, том 98, выпуск 5, 790-793

DOI: https://doi.org/10.4213/mzm10897

Использование Общероссийского математического портала Math-Net.Ru подразумевает, что вы прочитали и согласны с пользовательским соглашением http://www . mathnet.ru/rus/agreement

Параметры загрузки:

IP : 35.173 .219 .12

26 апреля 2023 г., 04:01:46

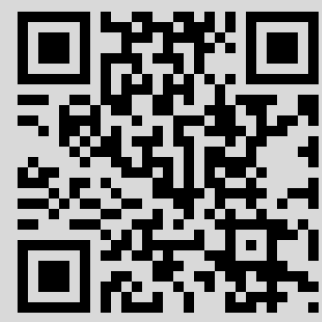




\section{Векторные расслоения конечного ранга на полных пересечениях конечной коразмерности в линейном инд-грассманиане}

\section{С. М. Ермакова}

1. Введение. Теорема Барта-Ван де Вена-Тюрина-Сато утверждает, что любое векторное расслоение конечного ранга на бесконечномерном комплексном проективном индпространстве $\mathbf{P}^{\infty}$ изоморфно прямой сумме линейных расслоений. Для расслоений ранга два эта теорема была доказана Бартом и Ван де Веном в [1], а для расслоений конечного ранга это было доказано Тюриным в [2] и Сато в [3]. В частности, теорема Барта-Ван де Вена-Тюрина-Сато верна для линейных инд-грассманианов и их линейных сечений [4]-[6].

В данной работе мы распространим эти результаты на случай полных пересечений в линейном инд-грассманиане. Основным полем является поле компексных чисел $\mathbb{C}$.

Вначале напомним определение линейных инд-многообразий, в том числе линейных инд-грассманианов, изученых Пенковым и Тихомировым [6], [7].

ОПРедЕЛЕНИЕ 1. Инд-многообразие $\mathbf{X}=\lim _{\longrightarrow} X_{m}$ определяется как прямой предел цепочки вложений

$$
\mathbf{X}:=\left\{X_{1} \stackrel{\phi_{1}}{\hookrightarrow} X_{2} \stackrel{\phi_{2}}{\hookrightarrow} \cdots \stackrel{\phi_{m-1}}{\hookrightarrow} X_{m} \stackrel{\phi_{m}}{\hookrightarrow \cdots\}} \cdots\right.
$$

где $X_{m}$ - гладкое алгебраическое многообразие для каждого $m \geqslant 1$.

Инд-многообразие $\mathbf{X}$ называется линейным, если на нем определен обратимый пучок

$$
\mathscr{O}_{\mathbf{X}}(1)=\lim _{\longleftarrow} \mathscr{O}_{X_{m}}(1)
$$

где для каждого $m \geqslant 1$ пучок $\mathscr{O}_{X_{m}}(1)$ обилен на $X_{m}$.

Пусть

$$
X_{m}=G\left(k_{m}, n_{m}\right), \quad \lim _{m \rightarrow \infty} k_{m}=\lim _{m \rightarrow \infty}\left(n_{m}-k_{m}\right)=\infty,
$$

и $\mathscr{O}_{X_{m}}(1)$ является образующей группы Пикара $X_{m}=G\left(k_{m}, n_{m}\right)$. Тогда инд-многообразие $\mathbf{X}$ называется линейным инд-грассманианом и обозначается $\mathbf{G}$.

Для линейного инд-грассманиана $\mathbf{G}$ определим плюккерово вложение $\mathbf{G} \hookrightarrow \mathbf{P}^{\infty}$ как прямой предел плюккеровых вложений грассманианов $G\left(k_{m}, n_{m}\right)$. Наконец, инд-гиперповерхностъю степени $d$ в $\mathbf{P}^{\infty}$ назовем прямой предел гиперповерхностей степеней $d$.

ОПредЕлениЕ 2. Рассмотрим линейный инд-грассманиан $\mathbf{G}$, вложенный по Плюккеру в $\mathbf{P}^{\infty}$. Пусть $\mathbf{Y}_{1}, \ldots, \mathbf{Y}_{l}$ - инд-гиперповерхности степеней $d_{1}, \ldots, d_{l}$ в $\mathbf{P}^{\infty}$. Линейное инд-многообразие

$$
\mathbf{X}=\mathbf{G} \cap \mathbf{Y}_{1} \cap \cdots \cap \mathbf{Y}_{l}
$$

называется полным пересечением в $\mathbf{G}$, если для всякого $m \geqslant 1$ многообразие

$$
G\left(k_{m}, n_{m}\right) \cap \mathbf{Y}_{1} \cap \cdots \cap \mathbf{Y}_{l}
$$

является полным пересечением.

Под векторным расслоением $\mathbf{E}$ ранга $r>0$ на $\mathbf{X}$ мы понимаем обратный предел $\mathbf{E}=$ $\lim _{E_{m}}$ цепочки векторных расслоений $\left\{E_{m}\right\}_{m \geqslant 1}$ ранга $r$, где $E_{m}$ - расслоение ранга $r$ на $X_{m}$ с фиксированными изоморфизмами $E_{m} \cong \phi_{m}^{*} E_{m+1}$.

Основным результатом настоящей работы является следующая теорема.

Теорема 1. Любое векторное расслоение $\mathbf{E}$ конечного ранга на полном пересечении $\mathbf{X} \subset \mathbf{G}$ конечной коразмерности изоморфно прямой сумме линейных расслоений.

2. Первоначальные сведения. Доказательство теоремы 1 опирается на понятия и результаты из статей [8] и [9].

DOI: $10.4213 / \mathrm{mzm} 10897$

(C) C. М. Ермакова, 
ОПРЕДЕЛЕНИЕ 3 . Пусть $X$ - проективное многообразие с обильным пучком $\mathscr{O}_{X}(1)$.

1. Назовем проективным подпространством в $X$ такое многообразие $M \simeq \mathbb{P}^{r}$ в $X$, что $\left.\mathscr{O}_{X}(1)\right|_{M} \cong \mathscr{O}_{\mathbb{P}^{r}}(1)$. В случае, если $M$ одномерно, назовем его проективной прямой в $X$, или просто прямой в $X$.

2. Путь $p_{n}(x, y)$ длины $n$ на $X$, соединяющий точки $x, y$,- это набор точек $x=x_{0}$, $x_{1}, \ldots, x_{n}=y$ в $X$ и набор проективных прямых $l_{0}, \ldots, l_{n-1}$ в $X$ таких, что $x_{i}, x_{i+1} \in l_{i}$.

Многообразие всех путей длины $n$, соединяющих точки $x$ и $y$, обозначим $P_{n}(x, y)$.

3. Назовем $X$ 1-связным, если для любых двух точек $x, y \in X$ существует путь, соединяющий $x$ с $y$.

Следующая теорема является следствием главного результата работы [8], основанного на формуле Монка для умножения в когомологиях пространства полных флагов.

Теорема 2. Пусть $k \leqslant[n / 2]$ u $X$ - полное пересечение грассманиана $G(k, n)$, вложенного по Плюккеру, с набором гиперповерхностей степеней $d_{1}, \ldots, d_{l}$ :

$$
X=G(k, n) \cap \bigcap_{i=1}^{l} Y_{i} .
$$

Eсли

$$
2 \sum_{i}\left(d_{i}+1\right) \leqslant \frac{k}{2} \leqslant\left[\frac{n}{4}\right],
$$

то многообразие $P_{k}(u, v)$ путей длины $k$, соединяющих любые две точки $u, v$ в $X$, непусто и связно.

ОПРедеЛЕНИЕ 4 . Пусть $\mathbf{E}$ - расслоение ранга $r$ на линейном инд-многообразии $\mathbf{X}$. Расслоение $\mathbf{E}$ назыается равномерным, если его ограничение на все проективные прямые имеет одинаковый тип расщепления. Расслоение $\mathbf{E}$ назыается линейно тривиалъным, если ограничение $\mathbf{E}$ на любую проективную прямую из $\mathbf{X}$ тривиально.

Теорема 3 [9]. Пусть $\mathbf{X}$ - полное пересечение в линейном инд-грассманиане $\mathbf{G}$. Всякое конечномерное векторное расслоение $\mathbf{E}$ на $\mathbf{X}$ равномерно.

Эта теорема является первым шагом в доказательстве расщепимости расслоения Е. Она выводится из теоремы Барта-Ван де Вена-Тюрина-Сато и свойств пространства проективных плоскостей в $\mathbf{X}$.

3. Флаг подрасслоений в Е. В этом разделе мы формулируем две теоремы и даем идеи их доказательств.

Теорема 4. Пусть $\mathbf{X}$ - полное пересечение в линейном инд-грассманиане $\mathbf{G}, \mathbf{E}$ - расслоение конечного ранга на $\mathbf{X}$. Тогда существует чепочка подрасслоений

$$
0=\mathbf{F}_{0} \subset \mathbf{F}_{1} \subset \cdots \subset \mathbf{F}_{s}=\mathbf{E}
$$

таких, что каждое фактор-расслоение $\mathbf{F}_{i} / \mathbf{F}_{i-1}$ для $1 \leqslant i \leqslant s$ является подкруткой линейно тривиального расслоения.

Объясним, как построить флаг подпространств в слое $\mathbf{E}_{x}$ расслоения $\mathbf{E}$ над точкой $x$ в $\mathbf{X}$. Для всякой проективной прямой $l$, проходящей через $x$, по теореме Гротендика в ограничении $\left.\mathbf{E}\right|_{l}$ имеется канонический флаг подрасслоений

$$
0=\mathbf{F}_{0} \subset \mathbf{F}_{1} \subset \cdots \subset \mathbf{F}_{s}=\left.\mathbf{E}\right|_{l} .
$$

Таким образом, в $\mathbf{E}_{x}$ возникает флаг подпространств. Пусть $B_{m}(x)$ - база семейства прямых на $X_{m}$, проходящих через $x$. В силу равномерности $\mathbf{E}$, сопоставляя прямой из $B_{m}(x)$ соответствующий флаг, мы получаем морфизм из $B_{m}(x)$ в пространство флагов в $\mathbf{E}_{x}$. Из следующей теоремы вытекает, что данный морфизм постоянен при больших $m$.

ПреДлОЖенИЕ. Для любого $d$ существует такое число $M(d)$, что для всякого $m>$ $M(d)$ всякий морфизм из $B_{m}(x)$ в многообразие размерности, менъшей $d$, постоянен. 
ИДЕЯ ДОКАЗАТЕЛЬСТВА. При больших $m$ многообразие $B_{m}(x)$ 1-связно [9; предложение 1]. Кроме того, всякая прямая из $B_{m}(x)$ содержится в проективном подпространстве $\mathbb{P}^{d} \subset B_{m}(x)$ большой размерности $d[9 ;$ лемма 3]. Таким образом, теорема сводится к факту, что всякий морфизм из $\mathbb{P}^{d}$ в многообразие меньшей размерности постоянен.

Мы объяснили, как выделить флаг в каждом слое расслоения Е. Для завершения доказательства теоремы 4 остается понять, что эти флаги образуют флаг подрасслоений. Расслоения $\mathbf{F}_{i} / \mathbf{F}_{i-1}$ являются подкрутками линейно тривиальных по построению.

После доказательства теоремы 6 теорема 1 будет вытекать из следующего результата.

Теорема 5. Пусть $\mathbf{X}$ - полное пересечение в линейном инд-грассманиане и $\mathbf{E}$ - расслоение конечного ранга на $\mathbf{X}$. Пусть

$$
0=\mathbf{F}_{0} \subset \mathbf{F}_{1} \subset \cdots \subset \mathbf{F}_{s}=\mathbf{E}
$$

- фллаг подрасслоений таких, что расслоение $\mathbf{F}_{i} / \mathbf{F}_{i-1}$ подкруткой тривиального расслоения на линейное расслоение для всякого $1 \leqslant i \leqslant s$. Тогда

$$
\mathbf{E}=\bigoplus_{i} \mathbf{F}_{i} / \mathbf{F}_{i-1}
$$

ИДЕЯ ДОКАЗАТЕЛЬСТВА. Покажем, что при больших $m$ ограничение векторного расслоения $\mathbf{E}$ на $X_{m}$ расщепляется в сумму $\bigoplus_{i} \mathbf{F}_{i} /\left.\mathbf{F}_{i-1}\right|_{X_{m}}$. Проведем индукцию по $i$. Пусть доказано, что

$$
\left.\mathbf{F}_{i-1}\right|_{X_{m}}=\sum_{1 \leqslant j \leqslant i-1} r_{j} \mathscr{O}\left(a_{j}\right) .
$$

Тогда $\left.\mathbf{F}_{i}\right|_{X_{m}}$ является расширением расслоения $\left.\mathbf{F}_{i-1}\right|_{X_{m}}$ при помощи $r_{i} \mathscr{O}\left(a_{i}\right)$. Чтобы доказать, что $\left.\mathbf{F}_{i}\right|_{X_{m}}$ расщепляется, достаточно знать, что

$$
H^{1}\left(r_{i} \mathscr{O}\left(-a_{i}\right) \otimes \sum_{1 \leqslant j \leqslant i-1} r_{j} \mathscr{O}\left(a_{j}\right)\right)=0 .
$$

Это равенство действительно выполняется для достаточно больших $m$ в силу теоремы Кодаиры, так как при больших $m$ многообразия $X_{m}$ являются многообразиями Фано и индекс $X_{m}$ стремится к бесконечности при $m$ стремящемся к бесконечности.

4. Критерий тривиальности линейно тривиальных расслоений. В этом пункте мы сформулируем теорему, которая дает критерий тривиальности линейно тривиальных расслоений.

Пусть $X$ - нормальное проективное многообразие, $E$ - векторное расслоение на нем. Пусть $Y$ - база семейства прямых на $X$. Пусть $Z \subset Y \times X$ - универсальная прямая. Обозначим $\pi$ и $p$ проекции из $Z$ в $Y$ и в $X$ соответственно. Отметим, что $\pi: Z \rightarrow Y$ есть $\mathbb{P}^{1}$-расслоение. Рассмотрим схему

$$
Z_{1}=Z \times_{Y} Z .
$$

Она параметризует прямые на $X$ с парой выделенных точек на них. Пусть $p_{1}, p_{2}: Z_{1} \rightarrow$ $X$ - композиции проекций $p_{r_{1}}, p_{r_{2}}: Z_{1} \rightarrow Z$ с отображением $p: Z \rightarrow X$. Далее мы определим индуктивно многообразие

$$
Z_{n+1}=Z_{n} \times_{X} Z_{1},
$$

с проекциями $p_{1}: Z_{1} \rightarrow X$ и $p_{n+2}: Z_{n+1} \rightarrow X$, посредством следующей диаграммы:

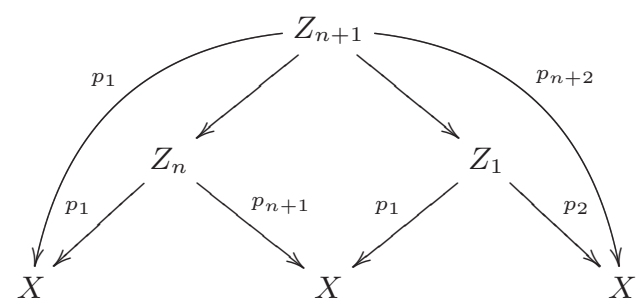


Для точки $x \in X$ положим

$$
Z_{n}(x):=p_{1}^{-1}(x) .
$$

Проекцию $Z_{n}(x) \rightarrow X$, индуцированную проекцией $p_{n+1}: Z_{n} \rightarrow X$, обозначим $f_{x, n}$.

Теорема 6. Предположим, что $X$ нормально и для некоторого $n>0$ и для некоторой точки $x \in X$ отображение $f_{x, n}: Z_{n}(x) \rightarrow X$ доминантно и имеет связные слои. Тогда любое линейно тривиальное векторное расслоение на $X$ тривиально.

Поясним идею, на которой основана эта теорема. Покажем как выбор базиса в векторном пространстве $E_{x}$ задает тривиализацию $E$ над $X$. Пусть $y$ - точка многообразия $X$, тогда многообразие $f_{x, n}^{-1}(y)$ является пространством $P_{n}(x, y)$ путей длины $n$, соединяющих $x$ и $y$. Так как $E$ тривиально на всех прямых из $X$, то для всякого пути $p_{n}(x, y)$ выбор базиса в $E_{x}$ задает базис в $E_{y}$. Таким образом, мы получаем отображение из $P_{n}(x, y)$ в пространство базисов в $E_{y}$. Так как первое многообразие проективно, непусто и связно по теореме 2, а второе афинно, то образ этого отображения - точка.

\section{5. Доказательство теоремы 1.}

ДоказАтеЛЬство. По теореме 4 в расслоении $\mathbf{E}$ есть флаг подрасслоений $\mathbf{F}_{i}$ таких, что фактор расслоение $\mathbf{F}_{i} / \mathbf{F}_{i-1}$ для $1 \leqslant i \leqslant s$ является подкруткой линейно тривиального расслоения.

Положим в теореме $6 X=X_{m}$ - полное пересечение в $G\left(k_{m}, n_{m}\right)$ и положим $n=k_{m}$. Тогда слоем отображения $f_{x, k_{m}}$ над точкой $y \in X_{m}$ является пространство путей длины $k_{m}$ на $X_{m}$, соединяющих $x$ и $y$. Положим в теореме $2 X=X_{m}$. Тогда для достаточно больших $m$ таких, что $k_{m}$ и $n_{m}$ удовлетворяют двойному неравенству

$$
2 \sum_{i}\left(d_{i}+1\right) \leqslant \frac{k_{m}}{2} \leqslant\left[\frac{n_{m}}{4}\right], \quad \text { где } \quad d_{i}=\operatorname{deg} \mathbf{Y}_{i},
$$

пространство путей $P_{k_{m}}(x, y)$ непусто и связно. Следовательно, все слои отображения $f_{x, k_{m}}$ непусты и связны; в частности, $f_{x, k_{m}}$ доминантно.

Таким образом, для $X_{m}$ выполняется условие теоремы 6 . Отсюда следует, что всякое линейно тривиальное векторное расслоение на $\mathbf{X}$ тривиально. А значит, каждое $\mathbf{F}_{i} / \mathbf{F}_{i-1}$ является подкруткой тривиального расслоения.

Наконец, применяя теорему 5, получаем, что расслоение $\mathbf{E}$ представимо в виде прямой суммы: $\mathbf{E}=\bigoplus_{i} \mathbf{F}_{i} / \mathbf{F}_{i-1}$. Теорема 1 доказана.

Автор выражает признательность своему научному руководителю А.С. Тихомирову, а также Дмитрию Панову и Александру Кузнецову.

\section{СПИСОК ЦИТИРОВАННОЙ ЛИТЕРАТУРЫ}

[1] W. Barth, A. Van de Ven, Classification of algebraic varieties and compact complex manifolds, Lecture Notes in Math., 412, Springer-Verlag, Berlin, 1974, 1-35. [2] А. Н. Тюрин, Изв. АН СССР. Сер. матем., 40:6 (1976), 1248-1268. [3] E. Sato, J. Math. Kyoto Univ., 17:1 (1977), 127-150. [4] J. Donin, I. Penkov, Int. Math. Res. Not., 34 (2003), 1871-1887. [5] I. Penkov, A. S. Tikhomirov, Algebra, Arithmetic, and Geometry: in Honor of Yu. I. Manin, Vol. II, Progr. Math., 270, Birkhäuser Boston, Boston, MA, 2009, 555-572. [6] И. Б. Пенков, А.С. Тихомиров, Матем. сб., 206:6 (2015), 49-84. [7] I. Penkov, А. S. Tikhomirov, Pure Appl. Math. Q., 10:2 (2014), 289-323. [8] C. М. Ермакова, Модел. и анализ информ. систем, 21:4 (2014), 35-46. [9] С. М. Ермакова, Модел. и анализ информ. систем, 22:2 (2015), 209-218. [10] R. Lazarsfeld, Positivity in algebraic geometry. I. Classical Setting: Line Bundles and Linear Series, Berlin, 2004.

\section{С. М. Ермакова}

Поступило

Ярославский государственный

28.04.2015

университет им. П.Г. Демидова

E-mail: svetlana.ermakova1802@gmail.com 